\title{
METÁSTASE CRANIANA DE ADENOCARCINOMA DE PRÓSTATA SIMULANDO MENINGIOMA PARASSAGITAL
}

\author{
J. FRANCISCO SALOMÃO* - YARA M. S. LIMA**-RENE D. LEIBINGER*** \\ ANTONIO C. FRANCO RIBAS**
}

RESUMO - Metástases osteoblásticas de câncer de próstata podem mimetizar meningiomas tanto do ponto de vista de radiologia simples, como de TC e de arteriografia cerebral. Os autores apresentam o caso de um paciente em que metástase craniana de adenocarcinoma prostático simulava meningioma parietal parassagital e chamam a atenção para a importância da correta interpretaçāo dos achados clinicos no dignóstico desta patologia.

\section{Cranial metastasiy from prostatic adenocarcinoma simulating parasagital meningioma.}

SUMMARY - The authors report the case of a 69 year-old man in whom X-Ray, CT and angiography findings typical for a parietal parasagital meningioma were caused by cranial metastasis from prostatic adenocarcinoma. The authors stress the importance of the correct interpretation of the clinical features in order to achieve a correct diagnosis.

Em 1953, Schaerer e Whitney 22 descreveram pela primeira vez metástase de carcinoma de próstata simulando meningioma. $\mathrm{Na}$ ocasião, os autores alertaram para o risco de se estabelecer erroneamente o diagnóstico de tumores tendo como base os chamados sinais patognomônicos e negligenciando aspectos discrepantes do quadro clinico.

A ocorrência de caso em que metástase craniana de adenocarcinoma de próstata simulava radiologicamente meningioma parassagital, constitui a base do presente relato.

\section{OBSERVAÇAO}

A.R.P., 69 anos de idade, branco, português, foi examinado inicialmente em 15-12-82 com queixa de dores na região dorsal e dificuldade progressiva para deambular iniciadas 5 meses antes. Na ocasiazo, o exame revelava paresia do membro inferior esquerdo, reflexos tendinosos simétricos e resposta plantar indiferente bilateralmente. $O$ estudo radiológico simples da coluna vertebral mostrou apagamento dos pedículos de C5 e T4 e o paciente foi encaminhado a exame urológico, descobrindo-se que a próstata estava aumentada de volume. Cintilografia óssea com TC $9 g$ revelou múltiplas éreas de aumento da atividade osteogênica em vértebras, arcos costais, lliaco e crânio; fosfatase ácida, $160 \mathrm{mU} / \mathrm{ml}, \mathrm{com}$ fraçăo prostática de $60 \mathrm{mU} / \mathrm{ml}$; urografia excretora compativel a tumor de próstata. $O$ paciente recusou a terapia proposta e năo mais foi visto até 21-02-83, quando foi admitido na Beneficência Portuguesa do Rio de Janeiro. Informavam os familiares que, ao longo dos últimos 30 dias, ele vinha se queixando de constante cefaleia e se tornara progressivamente apático nos dias que antecederam a internação. Ao exame neurologico, encontrava-se sonolento, confuso e atendendo com dificuldade aos comandos verbais; havia borramento blateral das papilas ópticas e as pupilas eram de lgual diâmetro, reagentes à luz; o dimfdio esquerdo encontrava-se parético com predominio crural e não havia comprometimento facial;

Serviço de Neurocirurgia, Hospital dos Servidores do Estado, INAMPS, Rio de Janeiro: * Chefe de Clínica e Neurocirurgiăo da Benefjcência Portuguesa, Rio de Janeiro; ** Ex-Residente; *** Neurocirurgiăo. 
a resposta plantar era em extensão à esquerda e os reflexos tendinosos estavam exaltados ipsilateralmente. O paciente sabia ser diabético há 4 anos. sendo controlado com dieta e hipcglicemiantes orais. Exames laboratoriais - glicemia, $134 \mathrm{mg} \%$; fosfatase alcalina, $100 \mathrm{mU} / \mathrm{ml}$; fosfátase ácida $160 \mathrm{mU} / \mathrm{ml}$, com fraçao prostática de $90 \mathrm{mU} / \mathrm{ml}$. Radiografia simples do crânio: extensa área de hiperdensidade óssea interessando à reguão parietal direita. Tomografia computorizada cerebral ('TC): lesão expansiva com altos valores de atenuaçăo, captante de contraste, ao nível dos lobos parietal e occipital direitos; colapso do ventrículo lateral direito e discreto desvio das estruturas da liuha média para o lado esquerdo; espessamento ósseo adjacente à lesão (Fig. 1). Arteriografia carotidea direita: massa homo-
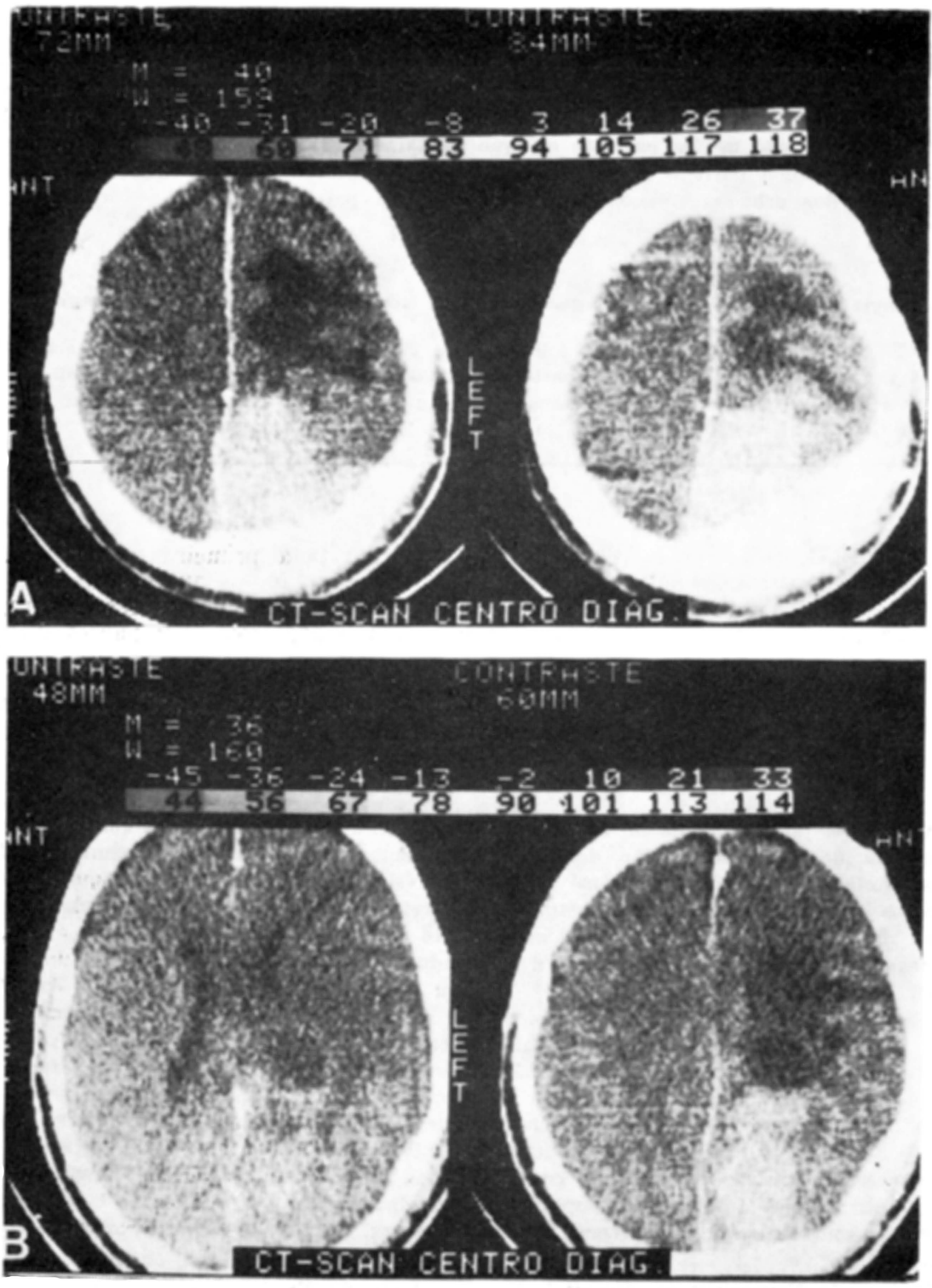

Fig. 1 - Caso A.R.P.: TC. Em A, massa com captação homogênea de contraste em localizacão parieto-occipital parassagital, aparentemente aderida à foice do cérebro; edema cerebral adjacente; note-se espessamento ósseo. Em $B$, deformacão do ventriculo lateral direito. 
gent גmente captante de oontraste, nutrida por ramos da carótida externa direita, com projeçấo na região parietal posterior. Cirurgia - Alguns dias após, o paciente foi suometido a exploração cirúrgica da lesão; o osso parietal encontrava-se amolecido e infiltrado por tumoraçāo de consistência firme e coloraçåo acastanhada; a massa ocupava a região parietal posterior, estendia-se ao occipital e em direção à linha média, deprimindo a dura máter e comprimindo secundariamente o cérebro; nāo havia infiltração das meninges. Anatomia patológica - Foram examinados vários fragmentos irregulares de tecido com áreas ora fríaveis, ora de consistência firme, de coloraçao pardacenta, em meio a esquírolas ósseas; microscopia: estrutura ossea infiltrada por neoplasia de linhagem epitelial constifuida de pequenos ácinos revestidos por células cúbicas de núcleos hipercorados e atípicos; diagnóstico: adenocarcinoma metastático. Evolução - O pós-operatório transcorreu sem anormalidades. Duas semanas após a alta neurocirúrgica, sobreveio quadro de pneumopatia aguda complicada por cetoacidose diabética e septicemia. O óbito ocorreu 8 dias aṕ́s o início do quadro infeccioso. Nảo se obteve permissão para a realização de necrópsia.

\section{COMENTARIOS}

O comprometimento do sistema nervoso no câncer de próstata é, em geral, consequência de compressão medular secundária a metástases vertebrais. Metástases disseminadas são características deste tipo de câncer e, em dois terços dos casos, acometem o esqueleto e linfáticos, sendo o terço restante distribuído por visceras como o figado e os pulmões 1. Metástases cranianas de câncer de próstata são consideradas infrequentes por Coppola e Salanga 9 e, segundo Saitoh e col.20, correspondem a $2 \%$ do total de metástases ósseas. Por sua vez, a ocorrência de metástases intracranianas $\dot{e}$ extremamente rara, sendo poucos os relatos encontrados na literatura $5,7,8,10,11,19$, 21,24,25. A incidência de metástases cerebrais é estimada entre 1,4 e $4,4 \%$ dos casos $8,20,21,25$ e, na maioria dos estudos compulsados, sua ocorrência é restrita a achados de necrópsia. Metástases intracranianas de carcinoma prostático podem eventualmente apresentar-se sob a forma de hemorragia intracerebral 5 , de hematoma subdural 7, ou mesmo de implantes em tumores intracranianos primários 6 . Admite-se que a disseminação metastática dos carcinomas de próstata se faça tanto a partir da neoplasia primitiva quanto đe focos secundários ósseos ou pulmonares, configurando, no último caso, o chamado efeito cascata $\mathbf{5 , 8 , 2 4 , 2 5}$. Acredita-se que, na ausểncia de metástases ósseas ou pulmonares, as células tumorais possam alcançar o segmento cefálico através do plexo venoso descrito por Batson 4,9,20,21,24,25.

Metástases de câncer de próstata podem, a exemplo de outros tumores2,3,13,14,18,22, simular meningiomas, tanto clínica como radiologicamente $11,12,15,16,17,22,23$. Revendo a literatura, Lippman e col.17 colecionaram 13 exemplos de metástases de carcinoma de próstata mimetizando meningiomas, aos quais adicionaram dois casos observados por eles. Embora alguns desses 15 casos sejam de metástases intracranias 11,25, a maioria é relacionada a comprometimento craniano geralmente ao nível do esfenóide ou da órbita 15,16,17,22,23. Metástase para a abóbada craniana simulando meningioma, a exemplo do caso por nós apresentado, foi descrita por Lippman e col.17 e parece tratar-se de ocorrência mais rara. As semelhanças radiológicas observadas podem ser explicadas pelos padrōes osteoblásticos das lesốes ósseas do carcinoma de próstata, que muitas vezes se assemelham à hiperostose vista nos meningiomas 16.17. Da mesma forma, na $\mathrm{TC}$, tante os meningiomas como as metástases de adenocarcinoma de próstata reforçam-se de maneira homogênea após a introdução de contraste 16 . 0 fato de as metástases cranianas receberem seu suprimento sanguíneo de ramos da carótida externa, a exemplo dos meningiomas, pode tornar eventuais diferenças menos evidentes ainda.

No caso por nós relatado, as evidências clinicas de carcinoma de próstata, a demonstração cintilográfica de lesões ósseas disseminadas e a existência de tumor intracraniano com rápida evolução, levaram à suposição de processo de natureza metastática. No entanto, os achados neurorradiológicos sugestivos de meningioma justificavam a exploração cirúrgica da lesão devido à possibildade de coexistência das duas patologias.

Finalizando, podemos concluir que: as caracteristicas radiológicas classicamente atribuilas aos meningiomas podem ser observadas em lesóes de outra natureza; a possibilidade de metástase de carcinoma de próstata deve ser considerada no diagnóstico diferencial dos meningiomas, em se tratando de adultos idosos do sexo masculino. 


\section{REHEHENCIAS}

1. Arnheim FK - Carcinoma of the prostate: a study of the post-mortem lindingis lil one hundred and seventy-six cases. I Urol 6i:5y9, 1948.

2. Asbi $\mathrm{CB}$, Bamnister CM, Turnbull 1W -- erimary cranial vauit lynphoma lnimicking a meningioma. Neurochirurgia (Stuttgart) $26: 130,1983$.

3. Atweh GF, Jabbour N - Intracranial solitary extraskeletal plasmacytoma ressembling meningioma. Arch Neurol 39:57, 1982.

4. Batson $O V$ - The function of the vertebral veins and their role in the spread of metastasis. Ann Surg 112:138, 1940.

5. Baumann MA, Holoye PY, Hoi H - Adenocarcinoma oi prostate presenting us brain metastasis. Cancer $54: 1723,1984$.

6 Bernstein KA, Grumet KA, Wetzel N - Metastasis of prostatic carcinoma to intracranial meningioma. J Neurosurg 58:774, 1983.

7. Bucci MN, Farhat SM - Metustatic adenocarcinoma of the prostate as a cause of subdural hematoma. I Urol 135:803, 1986.

8. Catane K, Kaufman J, West C, Merrin C, Tsukuda Y, Murphy GP - Brain metastasis trom prostatic carcinoma. Lancer $38: 2583,1976$.

9. Coppola RJ, Salanga VD - Metastatic prostatic adenocarcinoma to the temporal bone. Neurology $30: 311,1980$.

10. Delaney $P$ - Subdural metastases from prostatic adenocarcinoma (Letter). J Neurol Neurosurg Psychiat 46:186, 1983.

11. Fink LH - Metastasis of prostatic adenocarcinoma simulating a faix meningioma. Surg Neurol 12:253, 1979 .

12. Khan AU, Greene LF, Neault RW - Orbital metastasis from prostatic carcinoma. Int Surg $62: 465,1977$.

13. Khosia VK, Banerjee AK, Chopra JS - Intracranial actinomycoma with osteomyelitis simulating meningioma: case report. J Neurosurg 60:204, 1984.

14. Killebrew K, Krigman M, Mahley MS Jr, Scatiiff JH - Metastatic renal cell carcinoma mimicking a meningioma. Neurosurgery 13:430, 1983.

15. Kirkwood JR, Margolis MT, Newton TH - Prostatic metastasis of the base of the skull simulating meningioma en plaque. Am J Roentegenol 112:774, 1971.

16. Kwee IL, Nakada $T$, St John JN - Triple fossa metastasis of prostate cancer. Neurosurgery 13:584, 1983.

17. Lippman SM, Buzaid AC, Iacono RP, Steinbronn DV, Stanisic TH, Rennels MA, Yang PJ, Garewal HS, Ahmann FR - Cranial metastases from prostate cancer simulating meningioma: report of two cases and review of the literature. Neurosurgery 19:820, 1986.

18. Maleki M, Robitaile Y, Bertrand G - Atypical xanthoastrocytoma presenting as a meningioma. Surg Neurol 20:235, 1983.

19. Penley MW, Kim YC, Pribram HF - Subdural metastases from prostatic adenocarcinoma. Surg Neurol 16:131, 1981.

20. Saitoh H, Hida M, Shimbo T, Nakamura K, Yamagata J, Satoh T - Mietastatic patterns of prostatic cancer: correlations between sites and number of organs involved. Cancer $54: 3078,1984$.

21. Sarma DP, Godeau L - Brain metastasls from prostatic cancer. J Surg Oncol 23:173, 1983.

22. Schaerer JP, Whitney RL - Prostatic metastasis simulating intracranial meningioma: a case report. J Neurosurg 11:546, 1953.

23. Shapiro R, Janzen AH - Osteoblastic metastases to the floor of the skull simulating meningioma en plaque. Am J Roentgenol 81:964, 1959.

24. Smith VC, Kasdon DL, Hardy RC - Metastatic brain tumor from the prostate: two unusual cases. Surg Neurol 14:189, 1980.

25. Taylor HG, Lefkowitz M, Skoog SJ, Miles BJ, McLeod DG, Coggin JT — Intracranial metastases in prostate cancer. Cancer 53:2728, 1984. 